\title{
On the Impact of NB-IoT on LTE MBB Downlink Performance
}

\author{
Milutin Davidović, Igor Tomić, Dejan Drajić, and Zoran Čiča
}

\begin{abstract}
Internet of Things (IoT) has become a part of everyday life as well as industry and society. One of the main concerns of a mobile operator before implementing Io $\mathrm{T}$ in the mobile network is how much the IoT traffic will affect the performance of MBB users. In this paper, we present the simulation study that analyzes the impact of NB-IoT implementation on LTE MBB performance, based on one real network case considering the current status as well as the projected future traffic growth in the network.
\end{abstract}

Keywords - IoT (Internet of Things), MBB (Mobile Broadband), MTC (Machine Type Communications), NB (Narrow Band), RBG (Resource Block Group).

\section{INTRODUCTION}

$\mathrm{O}_{\mathrm{n}}^{\mathrm{N}}$ $\mathrm{NE}$ of the hot topics and challenges that one modern mobile operator is faced with nowadays is certainly the implementation of IoT for various types of use cases. Generally, IoT use cases can be classified into two main categories - critical and massive MTC.

Critical MTC (Fig. 1) is addressing the IoT use cases that require an ultra-reliable network, very low latency and very high availability (remote health care, surgery, industrial automation, Intelligent Transport Systems (ITS), real-time professional audio, etc.). In most of these cases next generation of networks $(5 \mathrm{G})$ is needed to meet such requirements.

On the other hand, massive MTC (Fig. 2) requirements are mainly focused on low cost and low energy (long life) of devices, small data volumes transmissions and massive number of devices in the network like: wearables (e-health), smart city/smart home, asset tracking (logistics), environmental monitoring and smart metering (smart building) and smart manufacturing (monitoring, tracking). Most of these applications can be addressed with

Paper received March 29, 2019; accepted May 08, 2019. Date of publication July 31,2019 . The associate editor coordinating the review of this manuscript and approving it for publication was Prof. Zorica Nikolić.

This paper is revised and expanded version of the paper presented at the 26th Telecommunications Forum TELFOR 2018 [12].

Milutin Davidović, Ericsson d.o.o, Milentija Popovica 5a, 11070, Belgrade, Serbia (e-mail: milutin.davidovic@ericsson.com)

Igor Tomić, Aspire Technology ULC, Vladimira Popovica 6, Unit B10, 11070, Belgrade, Serbia

(e-mail: igor.tomic@aspiretechnology.com).

Dejan Drajić, School of Electrical Engineering, University of Belgrade, Bulevar Kralja Aleksandra 73, 11000, Belgrade, Serbia (e-mail: ddrajic@etf.bg.ac.rs).

Zoran Čiča, School of Electrical Engineering, University of Belgrade, Bulevar Kralja Aleksandra 73, 11000, Belgrade, Serbia (e-mail: cicasyl@etf.bg.ac.rs). capabilities of LTE (Long Term Evolution) networks [1] - [3].

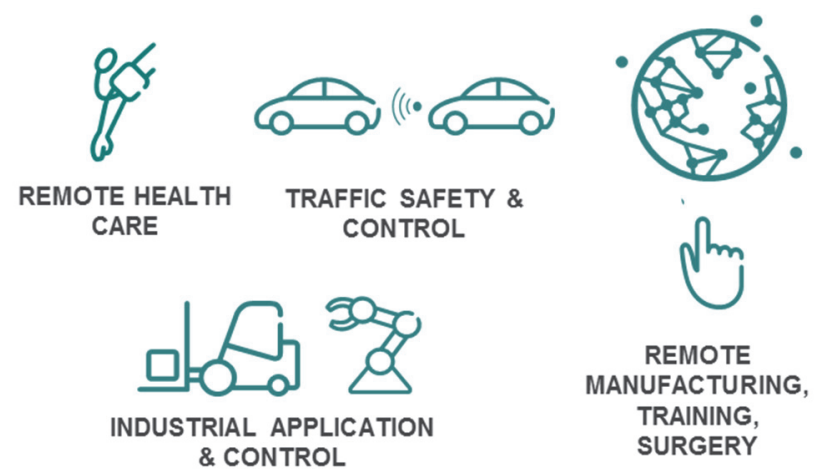

Fig. 1. Critical MTC.
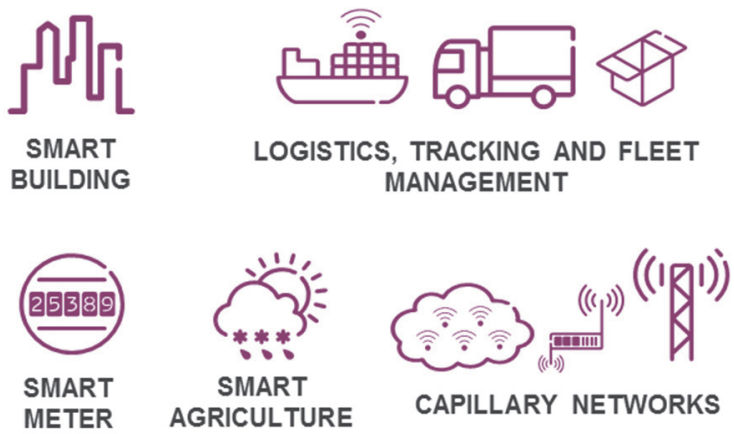

Fig. 2. Massive MTC.

In the following study, the focus is on massive MTC applications and main goal is to estimate the additional impact of NB-IoT deployment on existing LTE MBB performance, driven by coverage, capacity and system load [4] - [6]. This is done by taking the configuration and traffic load situation in one real LTE network and then simulating NB-IoT traffic over it.

As a simulation result, the estimation of the impact on LTE MBB and the level of performance degradation in terms of user experienced throughput decrease is given and commented for the specific cases depending on various factors such as MBB traffic, frequency band and bandwidth chosen for deployment of NB-IoT carrier. The paper is organized as follows: link budget and NB-IoT deployment options are considered in Section II, while in Section III modeling of NB-IoT in-band impact is given. The impact of NB-IoT on LTE MBB downlink performance is presented and analyzed in section IV, and section $\mathrm{V}$ concludes the paper and provides directions of the future work. 


\section{LINK BUDGET AND NB-IOT DEPLOYMENT OPTIONS}

Link budget is a powerful tool which can be used in various network performance estimations. By using theoretical and empirical models (environment, channel and wave propagation) and putting them together with load and empirical performance curves and configuration, the whole system can be successfully simulated (Fig. 3).

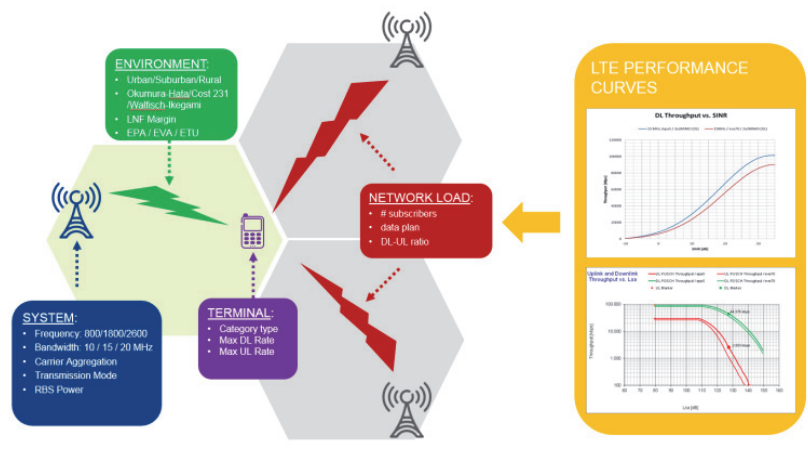

Fig. 3. General concept of Link Budget.

NB-IoT is an LTE-based narrowband radio access technology for the cellular internet of things. It provides greatly improved coverage, supports a massive number of low throughput devices, enables ultra-low device cost, and low device power consumption.

NB-IoT is a new 3GPP radio-access technology, but it is not fully backward compatible with existing 3GPP devices. It is however designed to achieve an excellent co-existence performance with legacy GSM, General Packet Radio Service (GPRS) and LTE technologies. NB-IoT reuses the LTE design extensively, including the numerologies, downlink Orthogonal Frequency-Division Multiple-Access (OFDMA), uplink Single Carrier Frequency-Division Multiple-Access (SC-FDMA), channel coding, rate matching, interleaving, etc.

What differs NB-IoT from for instance Cat-M1 (Cat-M1 operates at $1.4 \mathrm{MHz}$ bandwidth with a higher device complexity/cost than NB-IoT, while the wider bandwidth allows Cat-M1 to achieve greater data rates - up to $1 \mathrm{Mbps}$ ) is that NB-IoT aims to offer deployment flexibility allowing an operator to introduce NB-IoT using a small portion of its existing available spectrum. Another obvious difference is the fact that NB-IoT is clearly designed mainly targeting ultra-low-end IoT applications.

In essence, the following features and changes to legacy LTE characterize NB-IoT:

- New physical channels and signals

- Reduced peak rates

- $\quad$ Single receive antenna

- $\quad$ Reduced bandwidth of $180 \mathrm{kHz}$ (1 LTE PRB)

- New UE power class

- Enhanced coverage up to $20 \mathrm{~dB}$

- Half-duplex operation

NB-IoT supports 3 different modes of operation (deployment type):

- Stand-alone, where NB-IoT can replace one or more GSM carriers

- Guard band, utilizing the unused resource blocks within a LTE carrier's guard-band, and
- In-Band, utilizing resource blocks within a normal LTE carrier (Fig. 4).

Depending on the type of deployment, different effects of IoT traffic on MBB performance can be observed and modeled.

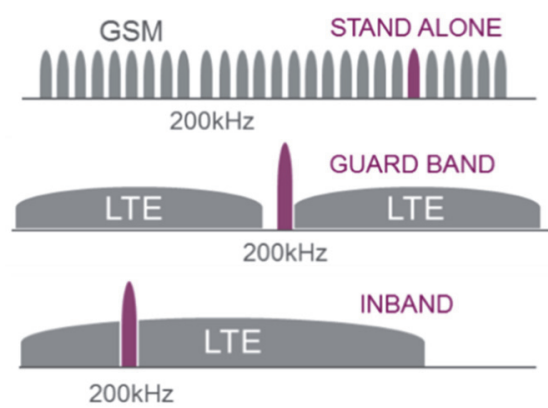

Fig. 4. NB-IoT deployment options.

Stand-alone NB-IoT is deployed with frequency reuse 1 in one frequency position in the GSM network. It requires $400 \mathrm{kHz}$ spectrum in total, i.e. $180 \mathrm{kHz}$ carrier bandwidth and $110 \mathrm{kHz}$ guard band on each side. Multiple NB-IoT carriers cannot be adjacent to each other per cell, but need $400 \mathrm{kHz}$ center-to-center separation. With regard to power and data rates, normal GSM output power of $20 \mathrm{~W}$ (43 dBm) makes high data rates possible.

Guard band deployment doesn't affect LTE MBB in terms of spectrum, as NB-IoT is placed in guard there is no reduction in the available number of resource blocks for legacy LTE. However, with guard band NB-IoT, possibilities to scale up capacity are limited, due to a limited guard band bandwidth. As near-far interference to nonupgraded LTE base stations can occur, a network-wide deployment is recommended. The guard band must contain the filter roll-off, and the loss from the filter roll-off increases the further out a carrier is placed. NB-IoT impacts LTE MBB performance, as power in amplifier is a common resource, and shared between LTE MBB and NB-IoT traffic. Boosting level, filter roll-off loss, number of carriers together affect power remaining for legacy LTE. As an example, assume $10 \mathrm{MHz}$ LTE with $40 \mathrm{~W}$ total power. Then, an NB-IoT output power of $3.2 \mathrm{~W}$ (35 dBm), using $6 \mathrm{~dB}$ power boosting, compared to LTE Power Spectral Density (PSD), gives around $0.4 \mathrm{~dB}$ reduction of maximum LTE power and ensures maintained total power.

In-band NB-IoT deployment impacts more LTE MBB performance as it affects spectrum and reduces available resource blocks for legacy LTE. Benefit is that NB-IoT capacity can easily be scaled up by simply adding more NB-IoT carriers. Alternatively, when there is no NB-IoT traffic, resources may be released for MBB traffic. As for guard band NB-IoT, network-wide deployment is recommended, due to potential near-far interference. Regarding the impact of NB-IoT on available power in amplifier, the same calculation for total power reduction in the guard band case is valid also in the in-band case.

Based on all above, the whole NB-IoT subsystem can be modeled and incorporated with the existing MBB simulation model.

However, two of these three deployment options, guard band and in-band, are related to LTE systems and therefore 
affect its performance.

As discussed above, guard band deployment has a minor impact on performance, slightly reducing the power per resource block in LTE legacy part as described. Therefore, in this study focus will be on the evaluation of the in-band deployment impact.

\section{MODELING OF NB-IOT IN-BAND IMPACT}

Starting point of this study is simulation of one LTE MBB network without NB-IoT implemented. The main assumptions are given in Table 1.

TABLE 1 MAIN ASSUMPTIONS AND INPUTS FOR LTE MBB NETWORK SIMULATION.

\begin{tabular}{|c|c|c|c|}
\hline \multirow{2}{*}{ Area } & Area covered & $\mathrm{km}^{2}$ & 311 \\
\hline & Number of sites & & 339 \\
\hline \multirow{2}{*}{ Traffic } & UL traffic & $\mathrm{GB} / \mathrm{BH}$ & 268 \\
\hline & DL traffic & $\mathrm{GB} / \mathrm{BH}$ & 2484 \\
\hline \multirow{4}{*}{ Environment } & Frequency & $\mathrm{MHz}$ & $1800 / 800$ \\
\hline & Bandwidth & $\mathrm{MHz}$ & $20 / 10$ \\
\hline & Environment & & Urban \\
\hline & Channel model & & EPA 5 \\
\hline \multirow{4}{*}{ Propagation } & Propagation model & & Walfisch-Ikegami \\
\hline & Walfisch-Ikegami H & $\mathrm{m}$ & 18 \\
\hline & Walfisch-Ikegami w & $\mathrm{m}$ & 20 \\
\hline & Walfisch-Ikegami b & $\mathrm{m}$ & 40 \\
\hline \multirow{7}{*}{$\begin{array}{l}\text { RBS (Radio } \\
\text { Base Station) }\end{array}$} & RBS type & & RRU \\
\hline & $\begin{array}{l}\text { RBS total power per } \\
\text { carrier }\end{array}$ & W & $2 \times 20 / 2 \times 40$ \\
\hline & TMA & & No \\
\hline & Sectors & & 3 \\
\hline & RBS antenna height & $\mathrm{m}$ & 25 \\
\hline & Antenna gain $(\mathrm{RBS}+\mathrm{UE})$ & $\mathrm{dBi}$ & 17.5 \\
\hline & Noise figure RU & $\mathrm{dB}$ & 1.6 \\
\hline \multirow{3}{*}{$\begin{array}{c}\text { UE (User } \\
\text { Equipment) }\end{array}$} & UE power & $\mathrm{dBm}$ & 23 \\
\hline & UE noise figure & $\mathrm{dB}$ & 7 \\
\hline & UE antenna height & $\mathrm{m}$ & 1.5 \\
\hline \multirow{3}{*}{ DL settings } & DL transmission mode & & $2 \times 2 \mathrm{MIMO}$ \\
\hline & DL modulation & & 64QAM \\
\hline & DL CRS antenna ports & & 2 \\
\hline \multirow{3}{*}{ UL settings } & UL transmission mode & & $1 \times 2$ SIMO \\
\hline & UL modulation & & 16 QAM \\
\hline & $\begin{array}{l}\text { P0 (Rx power target per } \\
\text { RB) }\end{array}$ & $\mathrm{dBm}$ & -80 \\
\hline \multirow{2}{*}{ Other losses } & $\begin{array}{l}\text { Body loss (where } \\
\text { applicable) }\end{array}$ & $\mathrm{dB}$ & 3 \\
\hline & Total penetration loss & $\mathrm{dB}$ & 16 \\
\hline $\begin{array}{l}\text { FDD/TDD } \\
\text { settings }\end{array}$ & Duplex & & FDD \\
\hline \multirow{3}{*}{$\begin{array}{l}\text { Advanced } \\
\text { settings }\end{array}$} & Scheduling strategy & & Resource fair \\
\hline & $\begin{array}{l}\text { Fractional pathloss - } \\
\text { alpha }\end{array}$ & & 0.8 \\
\hline & CRS boosting gain & $\mathrm{dB}$ & 0 \\
\hline $\mathrm{CCH}$ & pZeroNominalPucch & $\mathrm{dBm}$ & -117 \\
\hline \multirow{2}{*}{$\begin{array}{l}\text { LNF (Log- } \\
\text { Normal fading) }\end{array}$} & Coverage probability & & $90 \%$ \\
\hline & LNF stdev & $\mathrm{dB}$ & 10 \\
\hline
\end{tabular}

Based on these assumptions, simulations are done in Ericsson's internally developed tool for Link Budget and dimensioning purposes. This simulator is based on Ericsson's Coverage and Capacity Dimensioning [7] and Radio Wave Propagation [8] guidelines for LTE.

The simulation is performed for the real configuration and spectrum asset with a total capacity of 339 sites deployed in a target area $\left(311 \mathrm{~km}^{2}\right)$. Current MBB traffic load in Busy Hour and even load distribution between carriers is taken as an input from the real network as a reference. Traffic growth of $100 \%$ is also simulated in order to observe the effect of MBB traffic growth on the results. The Walfisch-Ikegami Urban $(\mathrm{H}=18 \mathrm{~m}, \mathrm{~W}=20 \mathrm{~m}$, $\mathrm{B}=40 \mathrm{~m}$ ) wave propagation model is used and adapted to the urban area of one European city which is taken as an example (Fig 5).

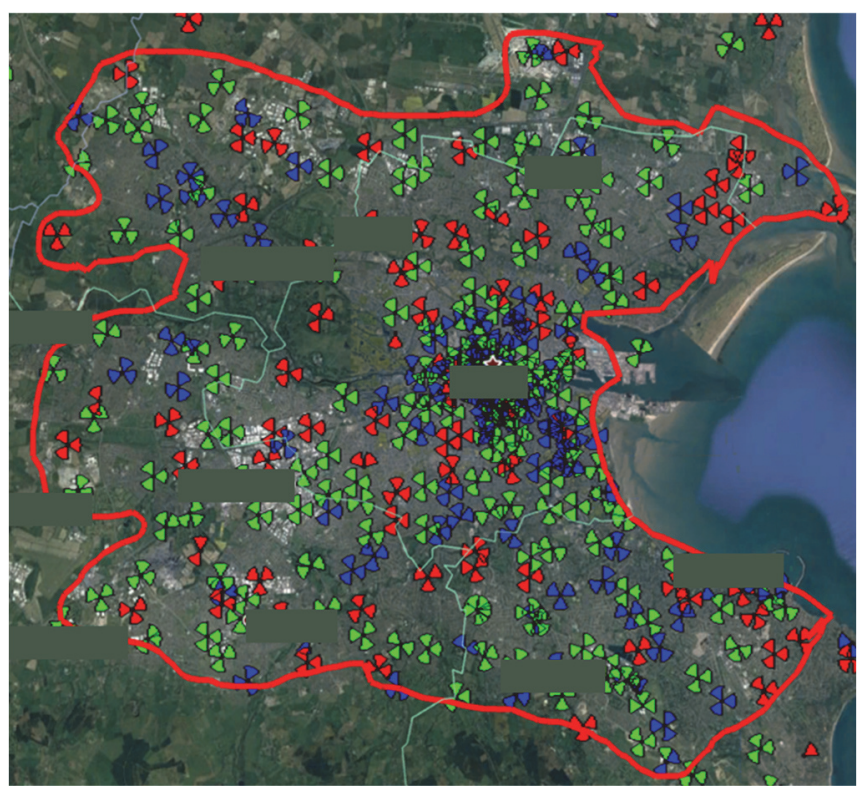

Fig. 5. Urban area of one European city with site distribution.

Deployment of different carriers in the network is considered (see Table 2) and therefore realistic traffic split (distribution) between carriers is considered in simulations. In this area, up to two LTE carriers are deployed. There are 267 sites with layer $1800 \mathrm{MHz}$ (L1800) and 228 sites with layer $800 \mathrm{MHz}$ (L800) in total. Around half of locations (156 sites) is deployed with two carriers (green sites in Fig. 5), while the rest are single-carrier sites -111 sites with only L1800 deployed and 72 sites with only L800 deployed.

TABLE 2 CARRIER DISTRIBUTION IN THE AREA.

\begin{tabular}{|c|c|c|c|}
\hline L1800 total & L800 total & \multirow{2}{*}{$\begin{array}{c}\text { Two carrier sites } \\
(L 1800+L 800)\end{array}$} & \multirow{2}{*}{$\begin{array}{c}\text { Total number } \\
\text { of sites }\end{array}$} \\
\hline 267 & 228 & & \\
\hline L1800 only & L800 only & \multirow{2}{*}{156} & \multirow{2}{*}{339} \\
\hline 111 & 72 & & \\
\hline
\end{tabular}

The second step is to model NB-IoT in band over a simulated LTE system and compare performance results to observe the impact of NB-IoT on LTE MBB performance. The main assumptions for NB-IoT model are shown in Table 3 (see next page). 
TABLE 3 MAIN ASSUMPTIONS AND INPUTS CONSIDERED FOR NB-IOT MODELING.

\begin{tabular}{|c|c|c|}
\hline MTC type & & NB-IoT \\
\hline Deployment options & & In-band \\
\hline Power boost & $\mathrm{dB}$ & 6 \\
\hline Noise figure UE & $\mathrm{dB}$ & 8.5 \\
\hline Extra coupling loss (EC) & $\mathrm{dB}$ & 10 \\
\hline DL utilization (fixed) & & $50 \%$ \\
\hline UL utilization (fixed) & & $50 \%$ \\
\hline Time-out timer & $\mathrm{s}$ & 10 \\
\hline Transaction model & & DoNAS \\
\hline Fraction of MTC devices with $\boldsymbol{E C}$ & & $10 \%$ \\
\hline DL overhead & & $30 \%$ \\
\hline UL overhead & & $3 \%$ \\
\hline
\end{tabular}

When it comes to defining the specific IoT use case, it is not relevant for this type of impact study (as downlink and uplink utilization is simulated as fixed $50 \%$ regardless of IoT use case). However, it is relevant for dimensioning of IoT carrier itself, which we will explore in our future work.

In terms of resources, NB-IoT system allocates one PRB for anchor carrier. However, as the current LTE DL scheduler (scheduler type 0 ) allocates resources in groups of PRBs - Resource Block Groups (RBG). RBG size is defined by 3GPP and it depends on system bandwidth (number of PRB in the bandwidth, $\mathrm{N}_{\mathrm{DL}}, \mathrm{RB}$ ) [9].

TABLE 4 LTE DL SChedUler Type 0 ResourCe ALLOCATION.

\begin{tabular}{|c|c|}
\hline $\begin{array}{c}\text { System Bandwidth } \\
\text { NDL, } \boldsymbol{R B}\end{array}$ & RBG Size \\
\hline$\leq 10$ & 1 \\
\hline $11-26$ & 2 \\
\hline $27-63$ & 3 \\
\hline $64-110$ & 4 \\
\hline
\end{tabular}

From capacity perspective, NB-IoT in general has a static (fixed) impact on resource consumption, not depending on the IoT load. In the observed network we have the spectrum asset of two carriers, $20 \mathrm{MHz}$ at $1800 \mathrm{MHz}$ band and $10 \mathrm{MHz}$ in $800 \mathrm{MHz}$ band, so activating one NB-IoT therefore takes:

- 4 PRBs in the case of $20 \mathrm{MHz}$

- 3 PRBs in the case of $10 \mathrm{MHz}$

This corresponds to the following overhead:

- $20 \mathrm{MHz}: 4 \%(4 / 100)$ instead if $1 \%(1 / 100)$

- $10 \mathrm{MHz}$ : $6 \%$ (3/50) instead of $2 \%(1 / 50)$

However, this overhead (unused PRBs in one RBG), can be used if there is a need for additional capacity defining non-anchor carriers which can carry additional traffic.

On the other hand, the power of these unused PRBs can be used for boosting the power of anchor carrier. Anchor NB-IoT carriers can be power boosted by $+6 \mathrm{~dB}$ by specification. The part of this additional power $(\Delta \mathrm{P} 1$ in Fig. 6) is taken from unused PRBs in NB. Remaining power $(\triangle \mathrm{P} 2$ in Fig. 6) is obtained by a slight reduction of all $\mathrm{RBs}$ in LTE legacy part.

However, the general impact also depends on the traffic/load status in MBB network.

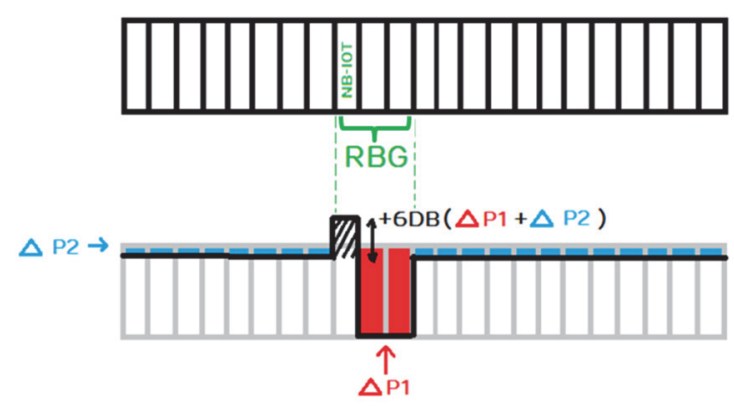

Fig. 6. NB-IoT Power Boosting.

\section{THE IMPACT OF NB-IOT ON LTE MBB DOWNLINK PERFORMANCE}

As previously stated, this study is focused on downlink. The impact on the average user-experience as well as on the cell edge performance in different layers/bands is observed and compared for different traffic load circumstances in the network. First, simulation is done for the current status of observed network in order to estimate how much potential NB-IoT deployment affects the current performance in the network (Fig. 7 and Fig. 8). Performance drop is defined as a user-experienced downlink throughput decrease after NB-IoT implementation, for both average throughput in a cell and throughput for user at cell edge.

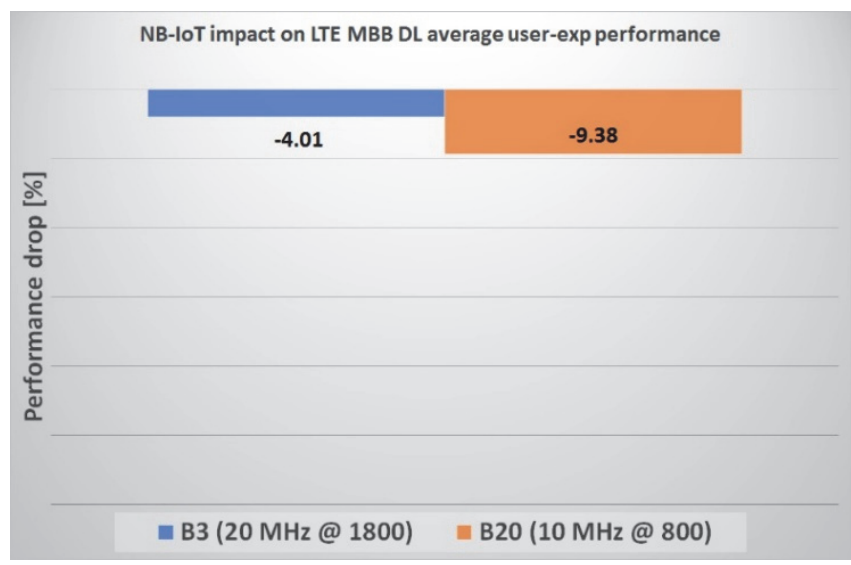

Fig. 7. NB-IoT In-Band deployment impact on DL average user-experience performance for current traffic load in LTE network.

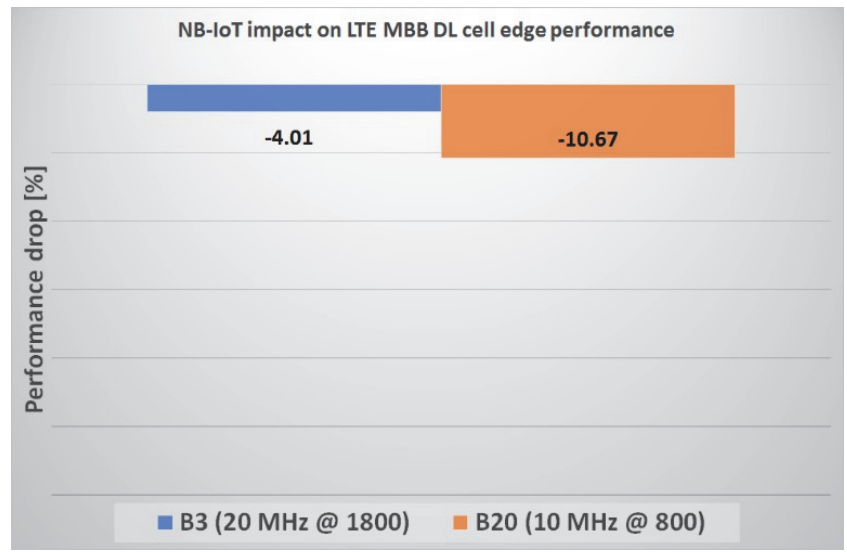

Fig. 8. NB-IoT In-Band deployment impact on DL cell edge performance for current traffic load in LTE network. 
Obtained results show a bit higher impact on cell edge compared to average user-experience in the cell, especially in $\mathrm{B} 20$ (800 $\mathrm{MHz}$ band), but in general the performance drops of about $4 \%$ in B3 (1800 MHz band) and 9-11\% in B20 can be expected. B20 is more impacted mainly due to less bandwidth deployed and therefore higher DL PRB utilization.

Naturally, the level of performance drop should increase as the traffic in network grows. In order to quantify this expectation, simulating the traffic growth of $100 \%$ is done, and results are shown in Fig. 9 and Fig. 10.

The traffic growth in LTE network results in the performance level drop that is not neglectable in both cases. The average user-experienced performance drop could go up to around $7.6 \%$ in B3 and $15.9 \%$ in B20.

For the users at the cell edge the performance could drop up to $9 \%$ in B3 and even up to $19 \%$ in B20.

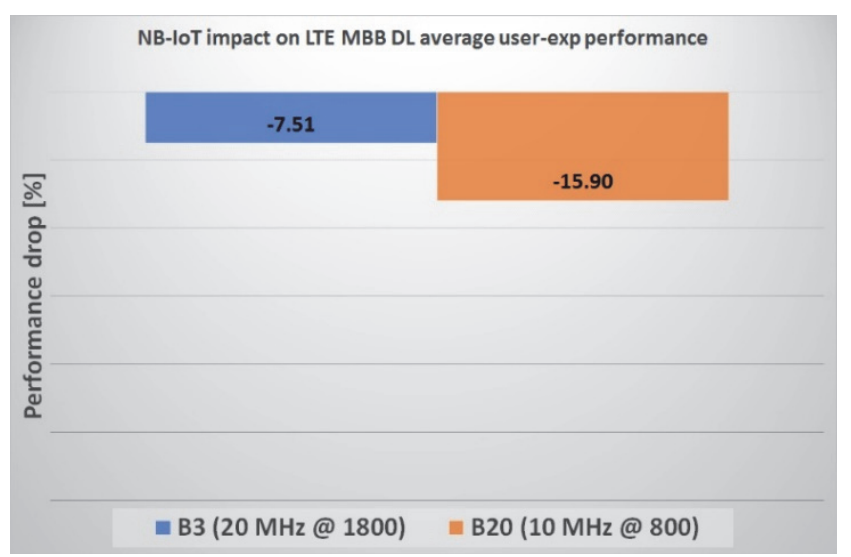

Fig. 9. NB-IoT In-Band deployment impact on DL average user-experience performance with $100 \%$ traffic growth in LTE network.

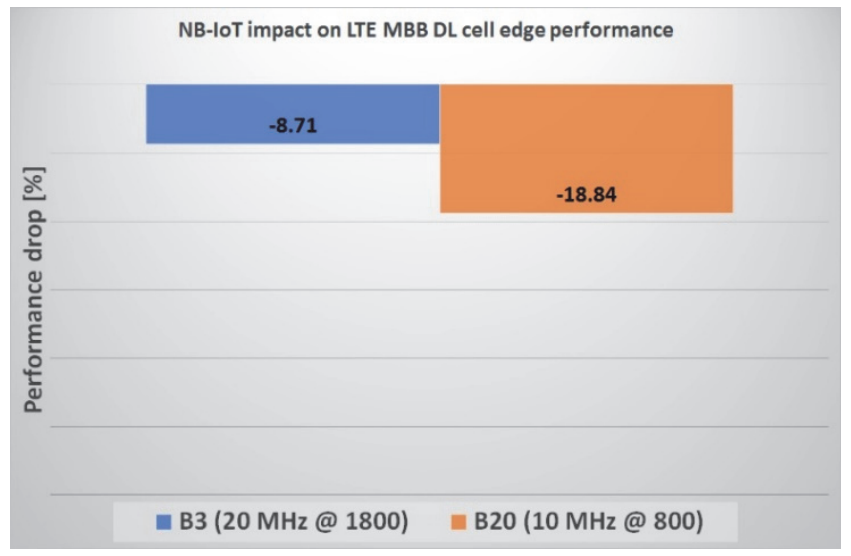

Fig. 10. NB-IoT In-Band deployment impact on DL cell edge performance with $100 \%$ traffic growth in LTE network.

\section{MAIN CONCLUSIONS AND WAYS FORWARD}

In this paper, IoT Link Budget analysis is used to anticipate its impact on LTE MBB performance. IoT traffic is modeled as fixed load (50\%), for complexity reasons and due to the fact that IoT traffic models are still immature. On the other hand, the load circumstances in NB-IoT carrier are almost not relevant as NB-IoT deployment allocates a fixed number of PRBs. In general, different interference rise is expected for different IoT traffic, but the main impact on performance actually comes from resource (PRB) allocation.

Generally, when it comes to LTE networks and NB-IoT, two possible deployment options could be observed Guard Band and In-Band. Deployment in Guard Band would have almost neglectable impact on MBB performance as it allocates resources out of LTE legacy bandwidth.

However, NB-IoT deployed inside LTE band will affect downlink MBB performance with a significant level of degradation mainly depending on the load circumstances in the network as it is shown in this study. Several conclusions can be made:

- Downlink impact is highly dependent on MBB load - it will increase as MBB load increases;

- Cell edge users will be affected more than the others;

- Higher impact on B20 mainly due to less bandwidth deployed and therefore higher DL utilization.

As a way forward, IoT traffic profiles for specific use cases can be defined and dimensioning of NB-IoT system can be done, observing IoT system performance and capacity. Another important step should be analyzing the uplink behavior and impact which would together with downlink give the complete picture and understanding of IoT impact on $\mathrm{MBB}$.

\section{REFERENCES}

[1] S. Lanndstorm, J. Bergstorm, E. Westerberg, D. Hammarwall, "NBIoT, A Sustainable Technology for Connecting Billions of Devices," Ericsson Technology Review, April 2016

[2] A. Rico-Alvarino, M. Vajapeyam, H. Xu, X. Wang, Y. Blankenship, J. Bergman, T. Tirronen, and E. Yavuz, "An overview of 3GPP enhancements on machine to machine communications," IEEE Communications Magazine, vol. 54, no. 6, pp. 14-21, June 2016.

[3] Evolved Universal Terrestrial Radio Access (E-UTRA) and Evolved Universal Terrestrial Radio Access Network (EUTRAN); Overall description (3GPP TS 36.300), 2016.

[4] M. Davidovic, S. Bjekovic, I. Tomic, "On the impact of network load on LTE network downlink performance", ETRAN 2015, Srebrno Jezero 2015

[5] I. A. Tomić, M. S. Davidović and S. M. Bjeković, "On the downlink capacity of LTE cell," 2015 23rd Telecommunications Forum Telfor (TELFOR), Belgrade, 2015, pp. 181-185.

[6] N. Gospić, I. Tomić, D. Popović and D. Bogojević, "Razvoj mobilnih telekomunikacija, od GSM do LTE," Univerzitet $u$ Beogradu, Saobraćajni fakultet, Beograd 2010.

[7] Coverage and Capacity Dimensioning, Ericsson Internal, 2016

[8] Radio Wave Propagation Guidelines, Ericsson Internal, 2016

[9] Evolved Universal Terrestrial Radio Access (E-UTRA); Physical layer procedures (3GPP TS 36.213, 7.1.6 Resource allocation), 2015

[10] M. Lauridsen, I. Z. Kovacs, P. Mogensen, M. Sorensen and S. Holst, "Coverage and Capacity Analysis of LTE-M and NB-IoT in a Rural Area," 2016 IEEE 84th Vehicular Technology Conference (VTCFall), Montreal, QC, 2016, pp. 1-5.

[11] Mobile IoT in the 5G future, NB-IoT and LTE-M in the context of 5G, GSMA report, April 2018.

[12] M. Davidovic, I. Tomic, D. Drajic and Z. Cica, "On the Impact of NB-IoT on LTE MBB Downlink Performance," 2018 26th Telecommunications Forum (TELFOR), Belgrade, 2018, pp. 1-4. 